\section{Thirty Years of Followup in 3 Patients with Familial Polyarteritis Nodosa due to Adenosine Deaminase 2 Deficiency}

\section{To the Editor:}

Three sisters of Northern European descent provided an opportunity to examine the longterm course and possible familial aspects of a rare disease, polyarteritis nodosa (PAN). Approval and consent was obtained from each patient.

The sisters have been cared for in the Johns Hopkins Vasculitis Center for the past 3 decades. At age 7, the first sister developed rash, fever, lethargy, and a deviated right eye and was treated empirically for tuberculosis. At age 8, these symptoms recurred along with abdominal pain, hypertension, and stroke. Renal angiography demonstrated microaneurysms and a skin biopsy confirmed the diagnosis of PAN. She was initiated on prednisone and azathioprine (AZA). Within the following year, she developed 2 bowel perforations with pathology demonstrating PAN and was treated with cyclophosphamide (CYC). By age 17, methotrexate was added for abdominal pain in the context of worsening mesenteric angiography. At age 19, she developed mononeuritis multiplex, and acute abdominal pain led to discovery of a ruptured renal aneurysm and upper gastrointestinal (GI) bleed, necessitating gastrectomy. She was treated with prednisone, CYC, and total parenteral nutrition. From ages 19 to 25 years, she had 8 hospitalizations for abdominal angina and GI bleeding and 1 for stroke. Angiography during this period showed 95-99\% stenosis of the celiac artery and diffuse changes of PAN in the superior and inferior mesenteric arteries as well as active skin lesions. Treatments included prednisone, mycophenolate mofetil (MMF), AZA, CYC, and ultimately etanercept (ETN). From age 25 to her current age of 44, while receiving ETN, she has had no hospitalizations for active PAN, and she has been able to work and travel.

The second sister developed fever, mononeuritis multiplex, and cutaneous vasculitis at age 7 . She did not respond to prednisone and was treated with AZA for 1 year. At age 8 she was transitioned to CYC but experienced persistent livedo reticularis. At age 23, she developed right eye blindness due to central retinal artery occlusion; the same condition occurred in the left eye the following year. From age 24 to her current age of 40 , she has not had ongoing PAN activity and has done well on treatment with prednisone, MMF, and ETN.

The third sister was diagnosed with PAN at age 6 when skin biopsy was performed in the context of livedo reticularis, subcutaneous nodules, and mononeuritis multiplex. She was treated with prednisone and briefly with AZA. Since diagnosis, she has done well on pentoxifylline aside from livedo reticularis that has been worse in the cold. She is currently age 41 and otherwise asymptomatic; thus treatment with tumor necrosis factor (TNF) inhibition has not been pursued.

None of the 3 sisters have developed hypogammaglobulinemia or hematologic abnormalities except for anemia due to GI bleeding in the first patient.

PAN is a systemic vasculitis of medium-sized vessels with an incidence of 4-10 per 100,000 individuals and an average age of onset of 40-60 years. Clinical manifestations may include systemic symptoms such as fever and weight loss and organ-specific findings that can include mononeuritis multiplex, cutaneous vasculitis, and aneurysms of the mesenteric and renal vasculature. The etiology of PAN is unknown, but hairy cell leukemia and infection with hepatitis B have been found to be associated with the condition ${ }^{1}$. While familial systemic vasculitis is generally quite rare, familial PAN has been identified in Israeli Jewish families in the Republic of Georgia $^{2}$. Exome sequencing performed in families with multiple members affected by PAN revealed that mutations in the CECR1 gene that codes for adenosine deaminase 2 (ADA2) were found in all patients with the disease. ADA2 exerts antiinflammatory effects in part through deactivation of extracellular adenosine, thus providing a biologic plausibility between deficiency of ADA2 in these patients and development of PAN ${ }^{2}$.

Given the early age of onset of PAN in these 3 sisters, they were referred for genetic testing (Figure 1). The 3 patients were found to be compound heterozygous with p.Asn423Lys/Exon7 deletion; the deletion was identified by multiplex ligation-dependent probe amplification. The parents are alive but have not been genetically tested. Measurement of ADA2 activity was performed using the ADA activity assay (Diazyme) by adding erythro-9-(2hydroxy-3-nonyl)adenine (Sigma-Aldrich) for inhibition of ADA1. All 3 patients were found to have decreased ADA2 enzyme activity compared to controls, supporting the diagnosis of familial PAN in the context of deficiency of ADA2.

This case series is instructive in several ways. First, it provides 3 decades of followup through which the natural history of a rare and newly recognized disease can be examined. Second, it demonstrates the wide spectrum of severity of familial PAN that provides evidence for variability of penetrance of expression of adenosine deaminase 2 deficiency. Finally, the response to TNF inhibition in the 2 siblings with more severe disease is consistent with reports in the literature of efficacy of this class of medication in treatment of this condition.

JASON LIEBOWITZ, MD, Rheumatology Fellow, Johns Hopkins Hospital; DAVID B. HELLMANN, MD, MACP, Aliki Perroti Professor of Medicine, Vice Dean, Johns Hopkins Bayview Medical Center, Johns Hopkins University School of Medicine, and Director, Department of Medicine, Johns Hopkins Bayview Medical Center, Baltimore; OSKAR SCHNAPPAUF, PhD, US National Human Genome Research Institute, National Institutes of Health, Bethesda, Maryland, USA. The authors thank Dr. Ivona Aksentijevich and Dr. Qing Zhou for their contributions to the genetic analysis of these patients. Address correspondence to Dr. J. Liebowitz, Johns Hopkins University, Division of Rheumatology, 5200 Eastern Ave., MFL Bldg., Center Tower, Suite 4100, Baltimore, Maryland 21224, USA.E-mail: jliebow3@jhmi.edu

\section{REFERENCES}

1. Ozen $\mathrm{S}$. The changing face of polyarteritis nodosa and necrotizing vasculitis. Nat Rev Rheumatol 2017;13:381-6.

2. Navon Elkan P, Pierce SB, Segel R, Walsh T, Barash J, Padeh S, et al. Mutant adenosine deaminase 2 in a polyarteritis nodosa vasculopathy. N Engl J Med 2014;370:921-31.

J Rheumatol First Release May 15 2019; 2019;46:8; doi: $10.3899 /$ jrheum. 180820 

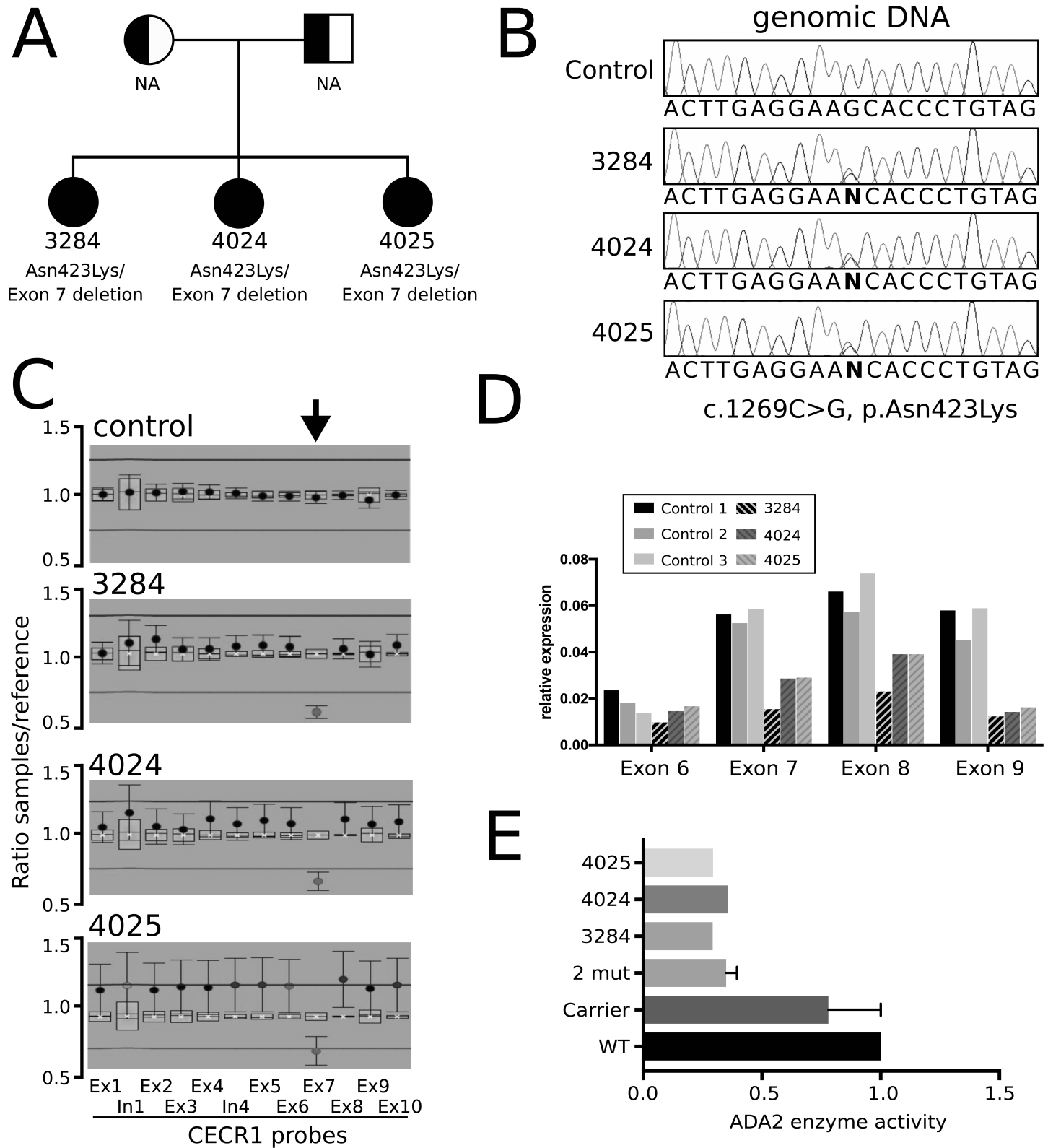

Figure 1. Exon7 Deletion family. A. Pedigree of the 2-generation family with affected patients 3284, 4024, and 4025. Squares indicate males, and circles represent females. Black and white symbols represent affected and unaffected individuals, respectively. Half-filled symbols indicate carrier individuals. Patients 3284, 4024, and 4025 were compound heterozygous for CECR1: p.Asn423Lys and a deletion comprising CECR1 exon 7. Parents of patients were not available (NA) for genetic testing. B. Sanger sequencing validation studies in patients 3284, 4024, and 4025 and an unrelated control. Sanger sequencing confirmed the heterozygous mutation CECR1: p.Asn423Lys in patients 3284, 4024, and 4025. This mutation was not detected in the unrelated control. C. Multiplex ligation-dependent probe amplification analysis of the CECR1 locus in control and in patients 3284, 4024, and 4025. On the X axis, used CECR1 probes are depicted and on the $\mathrm{Y}$ axis the ratio of relative fluorescence units (RFU) of the specific CECRI probe and the RFU value of the reference probe are shown. Lowered dots indicate a significant reduction in genomic quantity and therefore imply a heterozygous deletion at the specific location. Patients 3284,4024 , and 4025 showed heterozygous deletion of CECR1 exon 7. D. Quantitative reverse transcription of CECR1 exons 6 through 9 in 3 healthy controls and in patients 3284, 4024, and 4025. CECR1 mRNA detection of exon 7 through 9 is reduced in patients compared to controls. Shown are the relative expression values normalized to GAPDH. E. Decrease in ADA2 enzyme activity in patient plasma compared to healthy controls and carriers. Reduced ADA2 enzyme activity in patients 3284,4024 , and 4025 compared to healthy control (WT) and monoallelic carriers of a pathogenic CECR1 mutation. ADA2 enzyme activity of DADA2 patients is shown (2 mut). Ex: exon; In: intron; ADA2: adenosine deaminase 2; DADA2: deficiency of adenosine deaminase 2. 